Article

\title{
Arabidopsis ABA Receptor RCAR1/PYL9 Interacts with an R2R3-Type MYB Transcription Factor, AtMYB44
}

\author{
Dekuan Li ${ }^{1,2}$, Ying Li ${ }^{1}$, Liang Zhang ${ }^{1}$, Xiaoyu Wang ${ }^{1}$, Zhe Zhao ${ }^{1}$, Zhiwen Tao ${ }^{1}$, \\ Jianmei Wang ${ }^{1}$, Jin Wang ${ }^{3}$, Min Lin ${ }^{3}$, Xufeng $\mathrm{Li}^{1}$ and Yi Yang ${ }^{1,2, *}$
}

1 Key Laboratory of Bio-Resources and Eco-Environment of Ministry of Education, College of Life Sciences, Sichuan University, Chengdu 610065, China;

E-Mails: lidekuan198621@126.com (D.L.); lyscholes@163.com (Y.L.); liang.zhang113@gmail.com (L.Z.); ccandy1021@163.com (X.W.); zhaozhe89@126.com (Z.Z.); scuzhiwen8508@126.com (Z.T.); wangjianmei@scu.edu.cn (Jia.W.); lixufeng0507@gmail.com (X.L.)

2 State Key Laboratory of Hydraulics and Mountain River Engineering, Sichuan University, Chengdu 610065, China

3 Biotechnology Research Institute, Chinese Academy of Agricultural Sciences/Key Laboratory of Crop Biotechnology, Ministry of Agriculture, Beijing 100081, China;

E-Mails:wjdsz@vip.sina.com (JinW.); linmin57@vip.163.com (M.L.)

* Author to whom correspondence should be addressed; E-Mail: yangyi528@scu.edu.cn; Tel./Fax: +86-28-8541-0957.

Received: 22 March 2014; in revised form: 5 May 2014 / Accepted: 5 May 2014 /

Published: 13 May 2014

\begin{abstract}
Abscisic acid (ABA) signaling plays important roles in plant growth, development and adaptation to various stresses. RCAR1/PYL9 has been known as a cytoplasm and nuclear ABA receptor in Arabidopsis. To obtain further insight into the regulatory mechanism of RCAR1/PYL9, a yeast two-hybrid approach was performed to screen for RCAR1/PYL9-interacting proteins and an R2R3-type MYB transcription factor, AtMYB44, was identified. The interaction between RCAR1/PYL9 and AtMYB44 was further confirmed by glutathione S-transferase (GST) pull-down and bimolecular fluorescence complementation (BiFC) assays. Gene expression analysis showed that AtMYB44 negatively regulated the expression of ABA-responsive gene $R A B 18$, in contrast to the opposite role reported for RCAR1/PYL9. Competitive GST pull-down assay and analysis of phosphatase activity demonstrated that AtMYB44 and ABI1 competed for binding to RCAR1/PYL9 and thereby reduced the inhibitory effect of RCAR1/PYL9 on
\end{abstract}


ABI1 phosphatase activity in the presence of ABA in vitro. Furthermore, transient activation assay in protoplasts revealed AtMYB44 probably also decreased RCAR1/PYL9-mediated inhibition of $\mathrm{ABI} 1$ activity in vivo. Taken together, our work provides a reasonable molecular mechanism of AtMYB44 in ABA signaling.

Keywords: ABA; signaling; RCAR1/PYL9; receptor; AtMYB44; ABI1

\section{Introduction}

As sessile organisms, plants cannot escape away from, but have to face environmental stresses such as heat, cold, drought, and high-salinity stresses [1,2]. The phytohormone abscisic acid (ABA) is the crucial regulator in plant response to these stresses [3]. ABA enhances the capacity of plants in response to stress conditions through inducing stomatal closure, changing gene expression and increasing the accumulation of osmo-compatible solutes $[3,4]$.

In the last several years, the core ABA signaling components have been well ascertained. The RCARs/PYR1/PYLs act as ABA receptors [5-8], and the type 2C protein phosphatases (PP2Cs) act as negative regulators [9], while the SNF1-related protein kinase 2 family (SnRK2s) act as positive regulators [10-12]. In the absence of $\mathrm{ABA}$, the PP2Cs interact with and dephosphorylate SnRK2s to repress their kinase activities $[3,13]$. In the presence of ABA, RCARs/PYR1/PYLs interact with PP2Cs and inhibit their phosphatase activities, allowing SnRK2s to be activated [3,13]. Then, activated SnRK2s directly phosphorylate transcription factors ABFs/AREBs or components of the machinery regulating stomatal aperture, leading to ABA responses $[3,13]$.

Besides these core ABA signaling elements described above, MYB transcription factors (MYBs) are also key components in ABA signaling [14]. For example, AtMYB44, an R2R3-MYB transcription factor, has been well characterized for their regulatory roles in response to ABA [15-18]. Interestingly, controversial issues occurred about these studies. Huang et al. [15] and Jaradat et al. [16] demonstrated that AtMYB44 negatively regulated ABA signaling, while Jung et al. [17], and Persak and Pitzschke [18] revealed that over-expression of AtMYB44 led to ABA hypersensitivity in Arabidopsis. In addition, AtMYB44 was shown to be phosphorylated by MPK3 [18,19], but how AtMYB44 participates in ABA signaling remains unknown.

In this study, we demonstrate that AtMYB44 interacts with the ABA receptor RCAR1/PYL9, and reduces its inhibitory effect on ABI1 activity. This finding indicates that AtMYB44 may act as a negative regulator in $\mathrm{ABA}$ signaling. To our knowledge, this is the first detailed report on the molecular mechanism of how AtMYB44 is involved in ABA signaling regulation.

\section{Results}

\subsection{Identification of RCAR1/PYL9-Interacting Proteins by Yeast Two-Hybrid Screening}

The yeast two-hybrid system provides an efficient means to characterize core components in ABA signaling. The ABA receptor RCAR1/PYL9 has been identified by this system using ABI2 as bait [5]. Here, we used the yeast two-hybrid system to identify proteins that interact with RCAR1/PYL9. 
As a result, several different types of proteins were identified (Table S1). Three of them are closely related members of MYBs, namely AtMYB44, AtMYB70 and AtMYB77 (Table 1). AtMYB44 was shown to be involved in ABA signaling [15-17]. Thus, in the present study, we mainly focused our study on the interaction between RCAR1/PYL9 and AtMYB44 and the significance of their interaction in ABA signaling based on the biochemical and molecular analysis.

Table 1. RCAR1-interacting R2R3-type MYB transcription factors obtained by yeast two-hybrid assay in Arabidopsis.

\begin{tabular}{ccccc}
\hline Clone & $\begin{array}{c}\text { Accession Numbers in } \\
\text { TAIR }^{\text {a }}\end{array}$ & $\begin{array}{c}\text { AA Sequence in } \\
\text { Library }\end{array}$ & $\begin{array}{c}\text { AA Sequence in } \\
\text { TAIR }^{\text {a }}\end{array}$ & $\begin{array}{c}\text { Numbers } \\
\text { of Clones }^{\text {of }}\end{array}$ \\
\hline AtMYB44 & AT5G67300 & $48-305$ & 305 & 3 \\
AtMYB70 & AT2G23290 & $68-144$ & 309 & 1 \\
AtMYB77 & AT3G50060 & $26-286$ & 301 & 1 \\
\hline
\end{tabular}

${ }^{a}$ TAIR, The Arabidopsis Information Resource.

To test whether the full-length AtMYB44 and RCAR1/PYL9 interact, additional yeast two-hybrid assays were performed. As is shown in Figure 1A, AH109 yeast cells co-transformed with the full-length AtMYB44 prey construct and RCAR1/PYL9 bait vector grew well on SD/-L-T-H-A (-His-Ade) medium and turned blue on SD/-L-T (+His+Ade) $+\mathrm{X}-\alpha-$ Gal medium compared with the control transformants, indicating that the full-length AtMYB44 indeed interacts with RCAR1/PYL9 in yeast. Considering that RCAR1/PYL9 is an ABA receptor and its conformation can be changed upon binding to ABA $[3,13]$, we analyzed whether ABA affected the interaction between AtMYB44 and RCAR1/PYL9. Yeast two-hybrid assay showed that ABA had no significant effect on the interaction between these two proteins (Figure 1A).

\subsection{Demonstration of the Physical Interaction between RCAR1/PYL9 and AtMYB44 in Vitro and in Vivo}

To further confirm the interaction between RCAR1/PYL9 and AtMYB44, we performed in vitro GST pull-down assays. In these analyses, recombinant GST or GST-tagged RCAR1/PYL9 proteins were used to pull down His-tagged AtMYB44 proteins. As a result, AtMYB44 was retained by GST-RCAR1/PYL9 but not GST, suggesting that RCAR1/PYL9 interacts with AtMYB44 in vitro (Figure 1B). Subsequently, BiFC assay was employed to test the interaction between RCAR1/PYL9 and AtMYB44 in planta. AtMYB44 and RCAR1/PYL9 were fused to the $N$ - and $C$-terminal region of YFP to form AtMYB44-YFP ${ }^{\mathrm{N}}$ and RCAR1-YFP ${ }^{\mathrm{C}}$, respectively, and the two constructs were co-transfected into Arabidopsis protoplasts. The empty vectors $\left(\mathrm{YFP}^{\mathrm{C}}\right.$ and $\mathrm{YFP}^{\mathrm{N}}$ ) in combination with AtMYB44-YFP ${ }^{\mathrm{N}}$ or RCAR1-YFP ${ }^{\mathrm{C}}$ were used as negative controls. As a result, YFP fluorescence was observed in the nucleus of the protoplast cells co-transformed by AtMYB44-YFP ${ }^{\mathrm{N}}$ and RCAR1-YFP ${ }^{\mathrm{C}}$, while the negative controls failed to yield any fluorescent signal (Figure 1C). This result suggests that AtMYB44 interacts with RCAR1/PYL9 in the nucleus of Arabidopsis cells. 
Figure 1. Physical interaction between AtMYB44 and RCAR1/PYL9 in vitro and in vivo. (A) Yeast two-hybrid assay. The interaction between AtMYB44 and RCAR1/PYL9 was determined by growth assay and $\mathrm{X}$ - $\alpha$-gal staining on the indicated medium. When indicated, the medium was supplemented with $10 \mu \mathrm{M}$ ABA. Dilutions $(0.1,0.01$, and 0.001) (left three panels) or dilution (0.1) (right panel) of saturated yeast cultures containing the indicated plasmids were spotted on the indicated plates, and photographs were taken after 3 days at $30{ }^{\circ} \mathrm{C}$. BD (for DNA binding domain) indicates pGBKT7 vector; $\mathrm{AD}$ (for transcription activation domain) indicates pGADT7 vector. Scale bars $=2.5 \mathrm{~mm}$; (B) GST pull-down assay. $5 \mu \mathrm{g}$ of recombinant His-AtMYB44 protein was incubated with GST or GST-RCAR1/PYL9 in GST affinity resin. The eluates were resolved by SDS-PAGE and blotted using anti-His antibody. The position of His-AtMYB44 is indicated by an arrow. CBB (Coomassie Brilliant Blue) staining indicates amounts of GST-tagged proteins. Three independent experiments were performed and similar results were obtained; and (C) BiFC assay in Arabidopsis protoplasts. The $N$-terminal half of YFP was fused to AtMYB44 (AtMYB44-YFP ${ }^{\mathrm{N}}$ ), and the $C$-terminal half of YFP was fused to RCAR1/PYL9 $\left(\mathrm{RCAR}_{1-Y F P} \mathrm{C}\right)$. The combinations of plasmids are shown on the left. Co-expression of AtMYB44-YFP ${ }^{\mathrm{N}}$ and $\mathrm{YFP}^{\mathrm{C}}$ or $\mathrm{YFP}^{\mathrm{N}}$ and RCAR1-YFP ${ }^{\mathrm{C}}$ was used as a negative control, respectively. The nuclei were stained by DAPI. Scale bars $=10 \mu \mathrm{m}$.

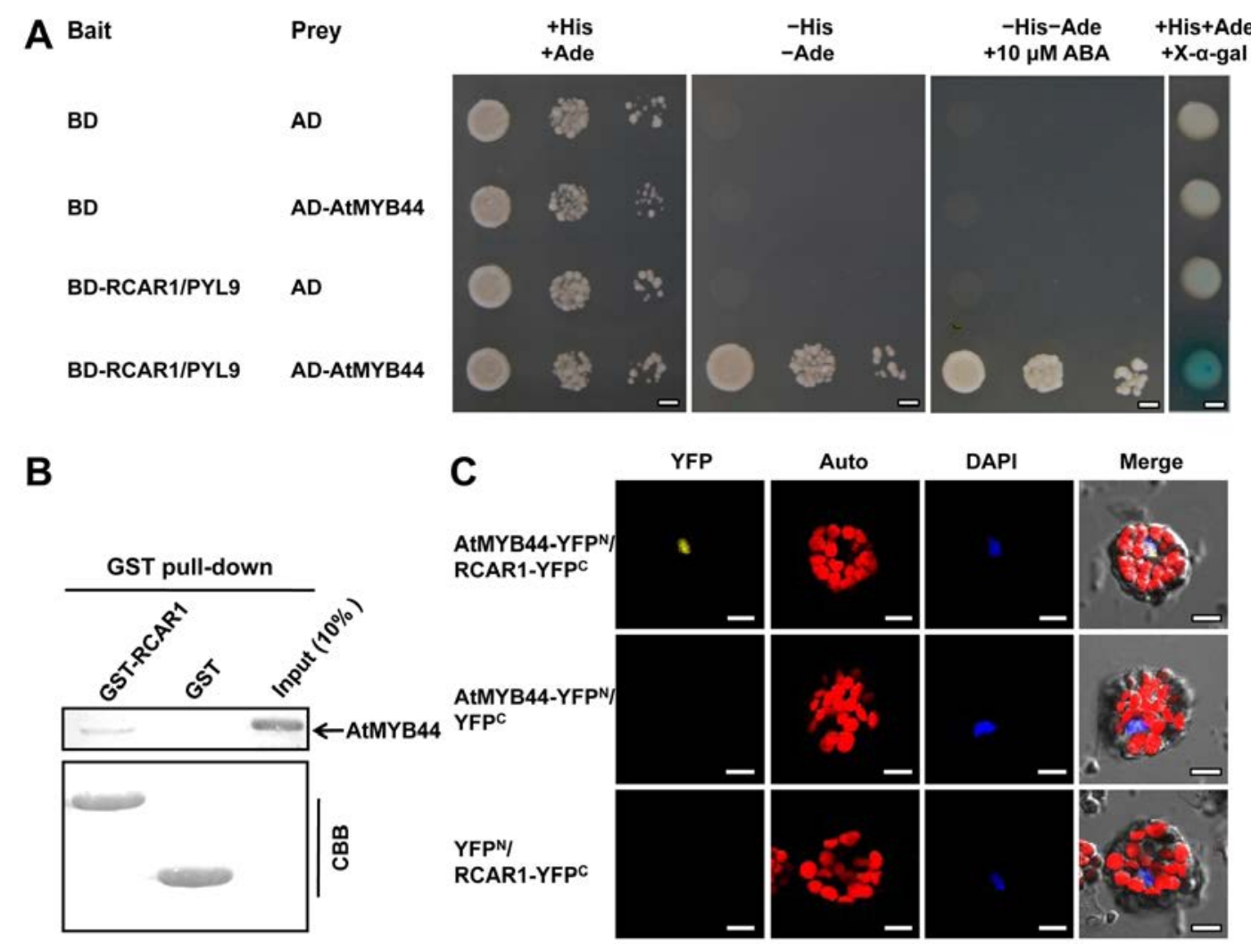

2.3. Analyses of the Interactions between RCARs/PYRI/PYLs and Other Members of the 22nd Subgroup of R2R3-MYBS

Considering that AtMYB44 belongs to the 22nd subgroup of R2R3-MYBs [20], we tested whether RCAR1/PYL9 also interacted with other members of the 22nd subgroup. Thus, AtMYB70, AtMYB73, 
AtMYB77 and AtMYB2 (as a control) were tested for their potential interactions with RCAR1/PYL9 in yeast two-hybrid assay. Figure 2A showed that RCAR1/PYL9 also interacted with AtMYB70, AtMYB73 and AtMYB77, but not with AtMYB2, a member of another subgroup of R2R3-MYBs, indicating that RCAR1/PYL9 may specially interact with the 22nd subgroup of MYBs. Similarly, ABA did not significantly affect the interactions between these proteins (Figure 2A).

Figure 2. Interactions between RCARs/PYR1/PYLs and the other members of 22nd subgroup of R2R3-MYBs. (A) Yeast two-hybrid assay was performed using RCAR1/PYL9 as bait and the full-length AtMYB70, AtMYB73, and AtMYB77 and AtMYB2 as preys. The empty BD and prey vectors were used as negative controls. Picture of the plates were taken after 3 days at $30^{\circ} \mathrm{C}$; and (B) Yeast two-hybrid assay was performed using the full-length RCAR3/PYL8, RCAR8/PYL5 and RCAR11/PYR1 as baits and AtMYB44 as prey. The empty AD and bait vectors were used as negative controls. Pictures of the plates were taken after 3 days at $30^{\circ} \mathrm{C}$. Scale bars $=2.5 \mathrm{~mm}$.



RCARs/PYR1/PYLs have been grouped into three different classes in Arabidopsis and RCAR1/PYL9 belongs to class I [5]. To test whether AtMYB44 also interacts with the other two classes of RCARs/PYR1/PYLs, RCAR3/PYL8, RCAR8/PYL5 and RCAR11/PYR1 were chosen for representative of different classes. Yeast two-hybrid assays demonstrated that AtMYB44 only 
interacted with RCAR3/PYL8, but not with RCAR8/PYL5 and RCAR11/PYR1 (Figure 2B), suggesting that AtMYB44 may specially interact with the 1st subclass of RCARs/PYR1/PYLs [5].

\subsection{AtMYB44 Negatively Regulates the Expression of ABA-Responsive Gene RAB18}

To understand the significance of the interaction between RCAR1/PYL9 and AtMYB44, we first investigated the role of AtMYB44 in ABA signaling. As mentioned above, there were controversial issues regarding the roles of AtMYB44 in the literature. In order to clarify these issues, we investigated the effect of a knockout mutation of AtMYB44 on the expression of RAB18, an important marker gene in ABA signaling [21,22], using a T-DNA insertion mutant of AtMYB44 (atmyb44) [15-17]. Analysis by quantitative reverse transcription-PCR (qRT-PCR) showed that expression of $R A B 18$ was slightly enhanced in the atmyb44 mutant compared to that in wild-type plants, both in the absence and presence of exogenous ABA (Figure 3A), indicating that AtMYB44 negatively regulates the expression of $R A B 18$.

To further test the effect of up-regulation of AtMYB44 on the expression of ABA-responsive gene $R A B 18$, we performed transient activation assays with Arabidopsis protoplasts using the $R A B 18$ promoter fused to the $F L U C$ gene ( $p R A B 18:: F L U C)$ as a reporter. In these assays, the effector genes, $A t M Y B 44-M y c$ or $A B I 1-G F P$, were expressed under the control of the 35S promoter and co-transfected together with the reporter construct into the protoplasts. Analysis of LUC activity demonstrated that co-transfection of $p R A B 18: \because F L U C$ with AtMYB44-Myc resulted in 1.5- and 4-fold reduced $p R A B 18:: F L U C$ expression in the absence and presence of $5 \mu \mathrm{M}$ ABA, respectively (Figure 3B). This suggests that AtMYB44 acts as a negative regulator in the expression of $R A B 18$ like ABI1, which is consistent with the result of microarray data published by Jaradat et al. [16].

\subsection{AtMYB44 and ABI1 Compete for Binding to RCAR1/PYL9}

To understand how AtMYB44 negatively regulates the expression of ABA-responsive genes, we further focused on the interaction between AtMYB44 and RCAR1/PYL9. Previous reports showed that competition might occur when two proteins interacted with the same protein. For example, the interaction between DELLAs and JAZs affects the binding of MYC2 to JAZs [23], and the interaction between SOS3 and SOS2 affects the binding of ABI2 to SOS2 [24]. Since RCAR1/PYL9 protein functions as an $\mathrm{ABA}$ receptor through interacting with $\mathrm{PP} 2 \mathrm{Cs}$ and inhibiting their phosphatase activities in the presence of ABA [5,6], the interaction between RCAR1/PYL9 and PP2Cs may affect the binding between RCAR1/PYL9 and AtMYB44. To test this possibility, we performed competitive GST pull-down assays. Recombinant GST or GST-AtMYB44 was used to pull down His-RCAR1/PYL9 in the absence or presence of His-ABI1 (one member of PP2Cs). In the presence of ABA, the interaction between His-RCAR1/PYL9 and GST-AtMYB44 was significantly impaired by an increased amount of His-ABI1 (Figure 4). In another assay, His protein was used as a control instead of His-ABI1. As is shown in Figure 4, His protein did not affect the binding of His-RCAR1/PYL9 and GST-AtMYB44. These results reveal that AtMYB44 and ABI1 compete in vitro for binding to RCAR1/PYL9 in the presence of ABA. To test whether other proteins that interact with RCAR1/PYL9 also compete with ABI1 for the binding to RCAR1/PYL9 in the presence of ABA, ArathEULS3 (one protein listed in Table S1) was representatively chosen for research. 
However, no significant competition between ABI1 and ArathEULS3 was found in our competitive pull down assay (data not shown), suggesting that not all proteins that interact with RCAR1/PYL9 can compete with ABI1 for the binding to RCAR1/PYL9.

Figure 3. AtMYB44 negatively regulates the expression of ABA-responsive gene $R A B 18$. (A) Relative expression levels of AtMYB44 and RAB18 in wild-type (Col) and atmyb44 mutant plants were determined by qRT-PCR analysis. 2-week-old seedlings were incubated in $1 \times$ MS liquid medium with ABA $(10 \mu \mathrm{M})$ or control solvent (DMSO) for $2 \mathrm{~h}$ before harvest. ACTIN2/8 served as an internal control. Error bars indicate $\operatorname{SD}(n=3)$. Three independent replicates were analyzed. Asterisks indicate the levels of statistical significance as determined by Student's $t$-test: * $p<0.05 v s$. Col; and (B) The effect of AtMYB44 on ABA-responsive $R A B 18$ gene expression was analyzed by transactivation assay in Arabidopsis protoplasts. Left panel: Schematic representation of reporter, effector and internal control constructs used in transactivation assays. Rc indicates Reporter construct; Ec indicates Effector constructs; Icc indicates Internal control construct; Right panel: AtMYB44 negatively regulates $R A B 18$ expression in protoplasts. pRAB18::FLUC, p35S::ABI1-GFP, and p35S::AtMYB44-Myc plasmids were co-transfected into Arabidopsis protoplasts from the wild-type plants as the indicated combinations. $R L U C$ was used as an internal control. After transfection, protoplasts were incubated for $5 \mathrm{~h}$ under light in the absence (open bars) or presence (filled bars) of $5 \mu \mathrm{M} \mathrm{ABA}$, and luciferase activity was measured. Values are mean $\pm \mathrm{SD}$ of three independent experiments.
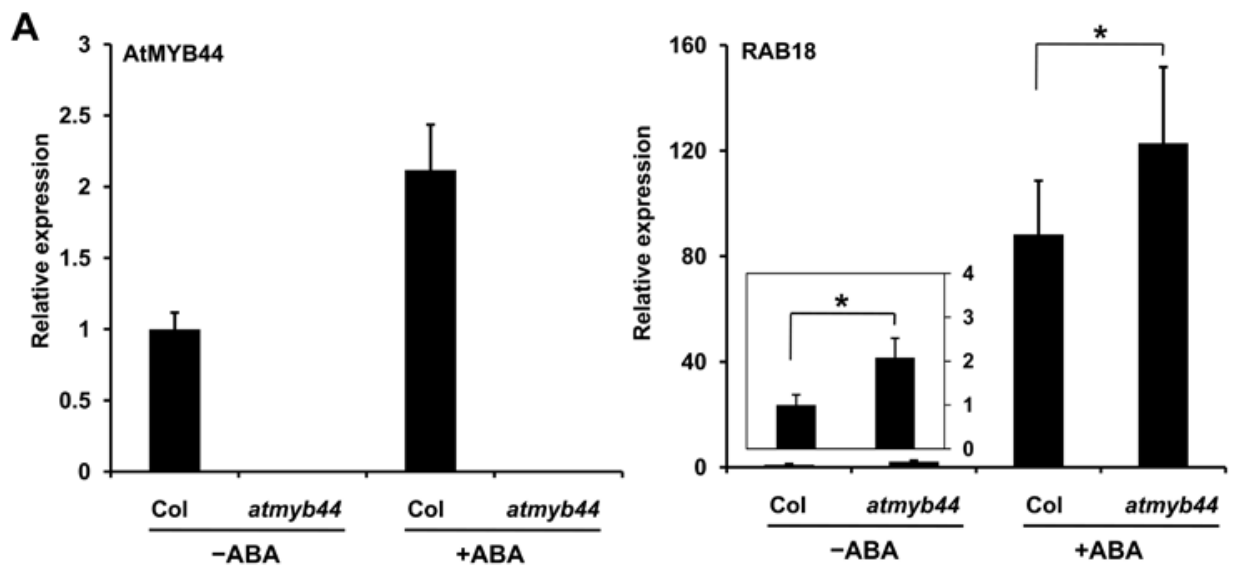

B
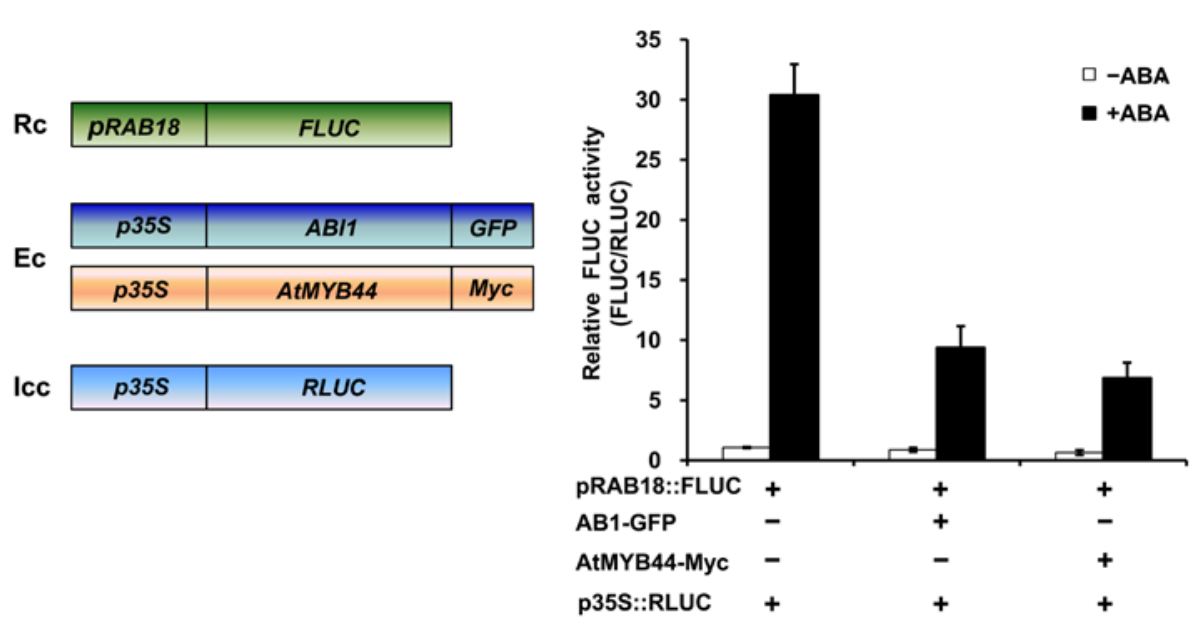
Figure 4. AtMYB44 competes with ABI1 for binding to RCAR1/PYL9 in vitro. His-RCAR1/PYL9 was pulled down with GST-AtMYB44 or GST in the absence or presence of His-ABI1 (or His). The gradient indicates increasing amount of His-ABI1. The immune-precipitated fractions were detected by western blotting with anti-His antibody (upper panel). GST-AtMYB44 input is shown in the lower panel. When indicated, the medium was supplemented with $10 \mu \mathrm{M}$ ABA. Three independent experiments were performed and similar results were obtained.

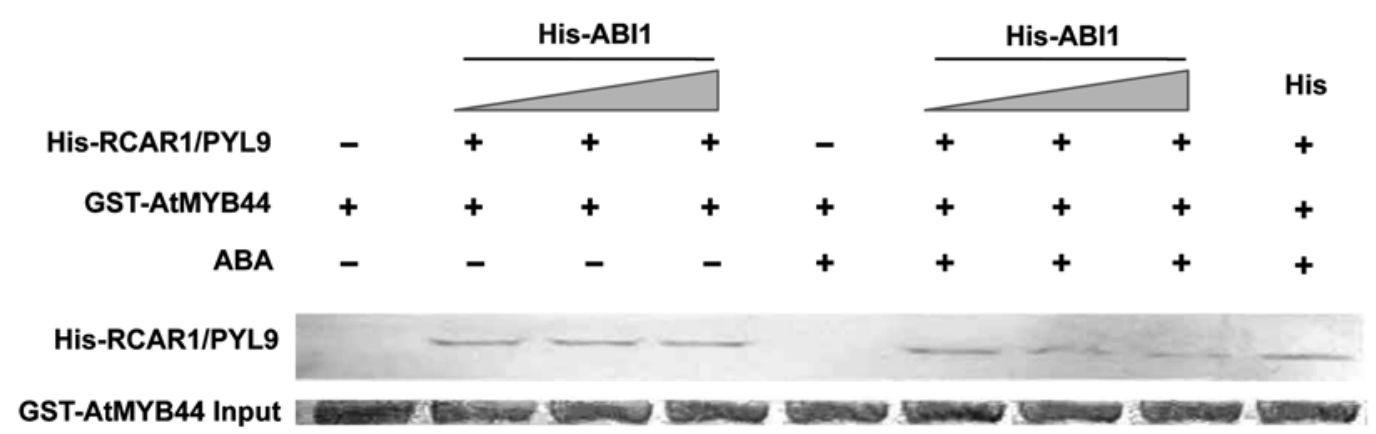

\subsection{AtMYB44 Probably Reduces the Inhibitory Effect of RCAR1/PYL9 on ABI1 Activity in Vitro and in Vivo}

Competition between AtMYB44 and ABI1 for binding to RCAR1/PYL9 led us to believe that AtMYB44 could facilitate releasing ABI1 from the ABI1-RCAR1/PYL9 complex through competing with the binding of RCAR1/PYL9 in the presence of ABA. To test this possibility, we examined the effect of AtMYB44 on RCAR1/PYL9-mediated inhibition of ABI1 phosphatase activity using recombinant His-ABI1, GST, GST-AtMYB44, and His-RCAR1/PYL9. As is shown in Figure 5, in the presence of ABA, AtMYB44 proteins alone did not affect the activity of ABI1, but RCAR1/PYL9 proteins alone significantly inhibited its activity as described [5]. When AtMYB44 proteins were added to the reaction mixtures of RCAR1/PYL9 and ABI1, the inhibitory effect of RCAR1/PYL9 on ABI1 phosphatase activity was significantly reduced in the presence of ABA rather than in the absence of ABA (Figure 5), suggesting that AtMYB44 interferes with the ability of RCAR1/PYL9 to suppress ABI1 phosphatase activity through competing for binding to RCAR1/PYL9 with ABI1 in the presence of ABA. To test whether other proteins that interact with RCAR1/PYL9 also affect RCAR1/PYL9-mediated inhibition of ABI1 activity in the presence of ABA, ArathEULS3 (one protein listed in Table S1) was representatively chosen for research. However, no significant effect was found in our PP2C enzyme assay (data not shown), suggesting that not all proteins that interact with RCAR1/PYL9 can reduce the RCAR1/PYL9-mediated inhibition of ABI1 activity. 
Figure 5. AtMYB44 reduces the inhibitory effect of RCAR1/PYL9 on ABI1 phosphatase activity in vitro. ABI1 phosphatase activity was determined using p-nitrophenyl phosphate (pNPP) as the substrate in the absence (open bars) or presence (filled bars) of $10 \mu \mathrm{M} \mathrm{ABA.}$ The concentrations of each protein component were $0.5 \mu \mathrm{M}$ for $\mathrm{ABI} 1,5 \mu \mathrm{M}$ for RCAR1/PYL9, and $10 \mu \mathrm{M}$ for GST-AtMYB44 or GST. The relative phosphatase activity of each reaction was normalized against the reaction containing substrate and ABI1 $(100 \%)$. Data are means \pm SD from three independent experiments.

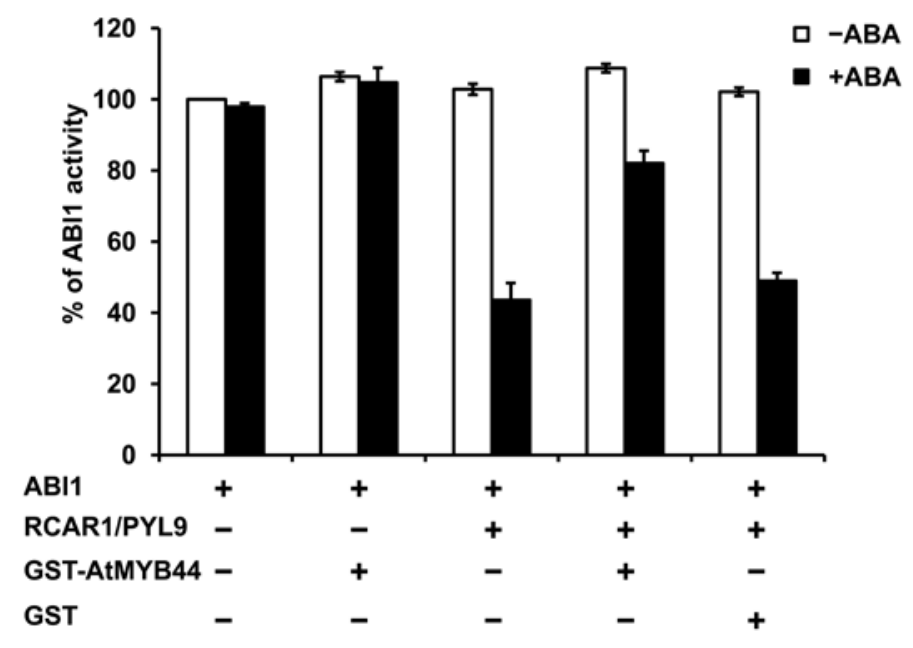

To determine the role of AtMYB44 in regulating the inhibitory effect of RCAR1/PYL9 on ABI1 phosphatase activity in vivo, we performed transient activation assay. Wild-type Arabidopsis protoplasts were co-transfected with $p R A B 18:: F L U C$ reporter construct and the indicated combinations of effector constructs encoding ABI1-GFP, RCAR1/PYL9-HA and AtMYB44-Myc. As is shown in Figure 6, transfection of $A B I 1$ significantly suppressed $p R A B 18:: L U C$ expression in the presence of exogenous $5 \mu \mathrm{M} A B A$, consistent with the role of ABI1 as a negative regulator of ABA-responsive genes [25,26]. Addition of RCAR1/PYL9 weakened the suppression of ABI1 on pRAB18::LUC expression, consistent with the result that RCAR1/PYL9 inhibits the phosphatase activity of ABI1 [5]. However, this antagonism is significantly reduced by further co-transfection of AtMYB44, indicating that AtMYB44 may also reduce the inhibitory effect of RCAR1/PYL9 on ABI1 in vivo. We noted that AtMYB44 alone also slightly increased ABI1-mediated inhibition of pRAB18::LUC expression (about 1.55-fold lower than without AtMYB44 in the presence of ABA), but its effect on antagonizing RCAR1/PYL9-mediated inhibition of ABI1 activity was more significant when $R C A R 1 / P Y L 9$ was co-expressed, where $p R A B 18:: L U C$ expression was about 2.66-fold lower than without AtMYB44 in the presence of ABA (Figure 6). This result suggests that AtMYB44 alone increasing $\mathrm{ABI} 1$-mediated inhibition of $p R A B 18:: L U C$ expression probably results from its inhibitory effect on endogenous RCAR1/PYL9-mediated inhibition of endogenous ABI1 activity in vivo. 
Figure 6. AtMYB44 likely reduces the inhibitory effect of RCAR1/PYL9 on ABI1 activity in vivo. (A) Schematic representation of reporter, effector and internal control constructs used in transactivation assay. Rc indicates Reporter construct; Ec indicates Effector constructs; Icc indicates Internal control construct; (B) AtMYB44 seems to reduce RCAR1/PYL9-mediated inhibition of ABI1 activity in protoplasts. pRAB18::FLUC, p35S::ABI1-GFP, p35S::RCAR1/PYL9-HA, and p35S::AtMYB44-Myc plasmids were co-transfected into Arabidopsis protoplasts from the wild-type plants as the indicated combinations. RLUC was used as an internal control. After transfection, protoplasts were incubated for $5 \mathrm{~h}$ under light in the absence (open bars) or presence (filled bars) of $5 \mu \mathrm{M} \mathrm{ABA}$, and luciferase activity was measured. Values are mean $\pm \mathrm{SD}$ of three independent replicates.


\section{Discussion}

AtMYB44 has been reported to participate in ABA signaling in the past few years [15-17], but the topic is controversial. In addition, although the function of AtMYB44 in ABA signaling has been elucidated [15-17], its interacting partners and detailed actions of its mechanism in ABA signaling remains largely unknown. In this study, AtMYB44 was identified as an RCAR1/PYL9-interacting protein via yeast two-hybrid screening. Effect of the functional RCAR1/PYL9-AtMYB44 interaction in ABA signaling was revealed by biochemical and molecular analysis. A hypothetical model for the action mechanism of AtMYB44 in ABA signaling is proposed in Figure 7. In the absence of ABA, RCAR1/PYL9 does not repress the phosphatase activity of ABI1, causing the active ABI1 to suppress the expression of ABA-responsive genes [3,5,13], while AtMYB44 interacts with RCAR1/PYL9 when it is over-expressed. In the presence of $\mathrm{ABA}, \mathrm{ABA}$ results in the inactivation of $\mathrm{ABI}$ and the 
expression of ABA-responsive genes [3,5,13]. However, when AtMYB44 is over-expressed, it competes with ABI1 for binding to RCAR1/PYL9, partly blocking the inhibitory effect of RCAR1/PYL9 on ABI1 and thus recovering the phosphatase activity of ABI1 to some extent and inhibiting the expression of ABA-responsive genes. Compared with prior work, we first identified the interaction between RCAR1/PYL9 and AtMYB44, further elucidated the significance of the interaction and proposed a possible model to explain how AtMYB44 is involved in ABA signaling. Our work can reasonably support the physiological observations of Jaradat et al. [16] and Ma et al. [5]. Of course, further genetic analysis still needs to be conducted in future studies to understand the biological roles of RCAR1/PYL9 and AtMYB44 more deeply.

Previous studies showed that all ABA receptors RCARs/PYR1/PYLs interact with PP2Cs and inhibit their phosphatase activities in the presence of ABA [5-7]. In the present study, we demonstrated that RCARs/PYR1/PYLs also interact with AtMYB44, which belongs to the R2R3-MYB transcription factors. Interestingly, unlike the interaction of RCARs/PYR1/PYLs with PP2Cs, RCAR1/PYL9 and RCAR3/PYL8 rather than RCAR8/PYL5 and RCAR11/PYR1 interact with AtMYB44, suggesting that AtMYB44 may specially interact with the 1st subclass of RCARs/PYR1/PYLs [5]. However, this result is contrary to the just published result of Jaradat et al., where they reported that AtMYB44 only interacts with RCAR3/PYL8 rather than the other members of PYR/PYL/RCAR family [16]. In fact, we found that RCAR2/PYL7 also interacts with AtMYB44 in the yeast two-hybrid assay (data not shown). These results indicate that although RCARs/PYR1/PYLs all act as ABA receptors, they still have different roles in planta. In addition, AtMYB70, AtMYB73 and AtMYB77, three homologous proteins of AtMYB44 in the 22nd subgroup of MYBs also interact with RCAR1/PYL9, but AtMYB2 [27], a member of the 20th subgroup of MYBs does not, indicating that RCAR1/PYL9 specially interacts with the 22nd subgroup of MYBs. This result also suggests that the four members of the 22nd subgroup of MYBs may share similar functions and therefore gene redundancy may exist. It can explain the potential redundancy of AtMYB44 with AtMYB77 in ABA responses and stress signaling [16]. Previous studies showed that the 22nd subgroup of MYBs contain two conserved motifs 22.1 and 22.2 [20]. Thus, it appears that RCAR1/PYL9 may physically bind to one (or both) of the motifs.

As mentioned before, several groups give the opposite opinions concerning the roles of AtMYB44 in ABA signaling [15-17]. In the present study, using qRT-PCR analysis and transient activation assays, we demonstrated that AtMYB44 negatively regulates the expression of ABA-responsive gene $R A B 18$, which is consistent with the previous result of microarrays [16]. Nevertheless, $R A B 18$ expression is only 1.2-1.5 times in atmyb44 mutant compared with that in the wild-type plants. Our explanation is that AtMYB44 has three homologous genes in Arabidopsis, including AtMYB70, $A t M Y B 73$ and $A t M Y B 77$, and therefore gene redundancy may exist. In fact, gene redundancy indeed exists, since double atmyb44 $\times$ atmyb77 mutants have more significant ABA-related phenotype than single atmyb44 and atmyb77 mutants [16]. In addition, we found that AtMYB70, AtMYB73 and AtMYB77 also interacted with RCAR1/PYL9, further suggesting that members in this family may share similar functions in ABA signaling. Based on our results and previous studies [16], we postulate that AtMYB44 negatively regulates the expression of ABA-responsive genes. However, the mechanism by which AtMYB44 negatively regulates ABA-responsive gene expression was undefined. In this study, we demonstrated that AtMYB44 reduces inhibitory effect of RCAR1/PYL9 on ABI1 
phosphatase activity in the presence of ABA. These results allow us to speculate that AtMYB44 negatively regulates the expression of ABA-responsive gene through decreasing the 1st subclass of RCARs/PYR1/PYLs-mediated inhibition of PP2Cs and thereby releasing phosphatase activity of PP2Cs in the presence of ABA. Accordingly, Huang et al. [15] and Jaradat et al. [16] has demonstrated that AtMYB44 negatively regulates ABA-mediated inhibition of seed germination and drought tolerance using a gain-of-function approach, which is similar to the role of PP2Cs, such as ABI1 [28]. However, in the present study, the interaction between AtMYB44 and RCAR1/PYL9 does not require exogenous ABA supplementation in vitro and in vivo. And ABA treatment does not affect their interactions in the yeast two-hybrid assay. In contrast, AtMYB44 reduces the inhibition of ABI1 activity by RCAR1/PYL9 in vitro only in the presence of ABA. This can be explained through the ABA-dependence of RCAR1/PYL9-mediated inhibition of PP2C. In fact, RCAR1/PYL9 hardly inhibits the phosphatase activity of ABI2 in the absence of exogenous ABA [5]. In addition, we must note that AtMYB44 appears to reduce the inhibitory effect of RCAR1/PYL9 on ABI1 activity both in the presence of $\mathrm{ABA}$ and in the absence of $\mathrm{ABA}$ in vivo, which is not completely consistent with the in vitro result. Our explanation is that despite exogenous ABA not being added, endogenous ABA is still at work in vivo. In fact, over-expression of $R C A R 1 / P Y L 9$ in protoplasts activating ABA signaling results from its response to endogenous ABA levels [5]. Further experiments are required to clarify this issue using ABA-deficient mutants [29]. Furthermore, concerning the role of the 22nd subgroup of MYBs, we did not rule out that R2R3 DNA-binding domains are necessary for their functions; perhaps they are necessary for their functions in other plant hormone signaling. In fact, R2R3 DNA-binding domains are necessary for AtMYB77 in auxin response [30]. However, the present study indicates that AtMYB44 interacts with the ABA receptor RCAR1/PYL9 and reduces its inhibitory effect on ABI1 activity to participate in ABA signaling. Accordingly, Fujii et al. have reconstituted an ABA signaling pathway and demonstrated that the RCARs/PYR/PYLs, PP2Cs (ABI1 is one of PP2Cs), SnRK2s and $\mathrm{ABF}$ are the only core components to complete the ABA regulation of gene expression [11]. Thus, the other proteins identified as being involved in ABA responses (for example, AtMYB44) may function to modulate the expression and/or activities of one or more of the core components. Considering the role of AtMYB44 in jasmonic acid (JA), salicylic acid (SA), ethylene (ET) and reactive oxygen species (ROS) signaling [31-33], and the interaction between AtMYB44 and RCAR1/PYL9, we are interested in testing if RCAR1/PYL9 is also involved in these signaling in future studies.

In addition, AtMYB44 is regulated by MPK3-mediated phosphorylation [18,19], though it seems that the phosphorylation state of AtMYB44 is not necessary for its function in ABA signaling, since wild-type AtMYB44 can interact with RCAR1/PYL9 and reduce its inhibitory effect on ABI1 phosphatase activity in vitro in our present study. Accordingly, DNA binding properties and localization of AtMYB44 have also been shown to be phosphorylation-independent [18]. This can explain why non-phosphorylatable, phosphomimetic and wild-type MYB44 over-expressing lines exhibited similar phenotypes in ABA-mediated inhibition of seed germination [18]. 
Figure 7. A hypothetical model for the action mechanism of AtMYB44 in ABA signaling. In the absence of ABA, AtMYB44 interacts with RCAR1/PYL9, but does not affect ABI1 activity. Upon ABA perception, AtMYB44 competes with ABI1 for binding to RCAR1/PYL9, releasing the inhibitory of RCAR1/PYL9 on ABI1 activity and thus down-regulating the expression of ABA-responsive genes. "*”" indicates that this component may also include RCAR2/PYL7, RCAR3/PYL8 and RCAR4/PYL10. “**” indicates that this component may also include AtMYB70, AtMYB73 and AtMYB77.

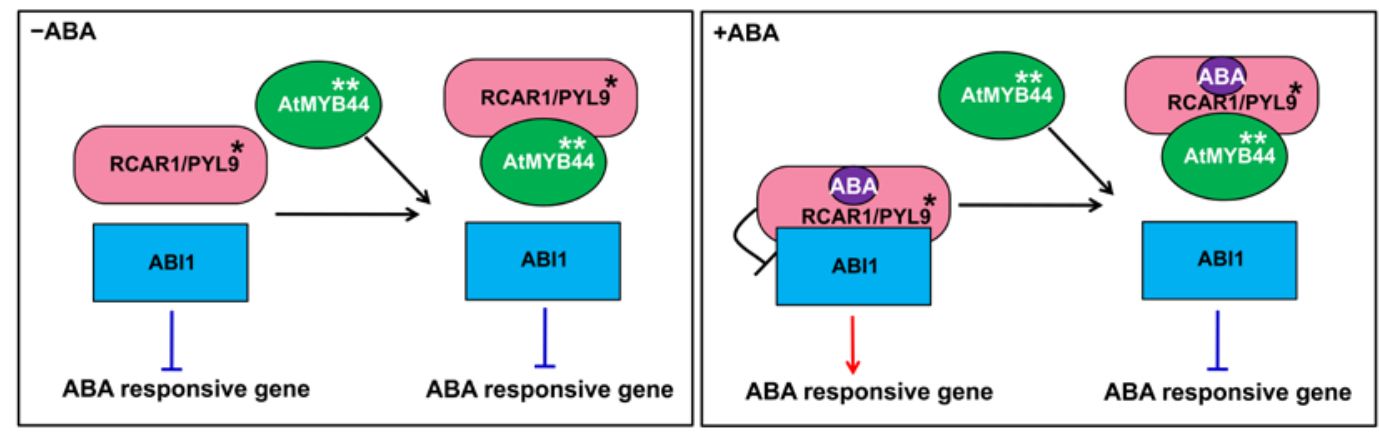

\section{Experimental Section}

\subsection{Plant Materials and Chemicals}

All Arabidopsis plants used in this work were in the Columbia (Col-0) background. The atmyb44 T-DNA insertion mutant (Salk_039074) has been previously described [15-17].

For in vitro cultivation, seeds were grown in soil-vermiculite mixtures at $22{ }^{\circ} \mathrm{C}$ under $60 \%$ humidity with cycles of $16 \mathrm{~h}$ light and $8 \mathrm{~h}$ dark. For plate culture, seeds were soaked in distilled water for 3 days at $4{ }^{\circ} \mathrm{C}$, then surface sterilized by ethanol and $\mathrm{HgCl}_{2}$. After surface sterilization, seeds were sown on the Murashige-Skoog (MS) medium [34] containing 3\% sucrose and 0.8\% agar, pH 5.9.

All chemicals used were of analytic grade or highest purity and purchased from Sigma (St. Louis, MO, USA), Amresco (Solon, OH, USA) and Chengdu Kelong Chemical Reagent Factory (Chengdu, China).

\subsection{Plasmid Construction}

For yeast two-hybrid assay, the ORFs of RCAR1/PYL9 and RCAR3/PYL8 were amplified and cloned into the EcoR I-Pst I sites of pGBKT7 from Clontech Laboratories, Inc. (Takara Bio Group, Otsu, Japan); the ORFs of RCAR8/PYL5 and RCAR11/PYR1 were amplified and cloned into the EcoR I-BamH I sites of pGBKT7; the ORFs of AtMYB44, AtMYB70, AtMYB73, AtMYB77 and AtMYB2 were amplified and cloned into the EcoR I-Xho I sites of pGADT7 from Clontech Laboratories, Inc. For GST pull-down assay and PP2C enzyme assay, the ORFs of RCAR1/PYL9 and AtMYB44 were amplified and subcloned into pGEX-2T (Amersham, Piscataway, NJ, USA), yielding pGEX-RCAR1/ PYL9 (cloning via EcoR I and Xho I sites) and pGEX-AtMYB44 (cloning via EcoR I and Xho I sites); the ORFs of RCAR1/PYL9, AtMYB44 and ABI1 were amplified and subcloned into pET28a (Novagen, Darmstadt, Germany), yielding pET28A-RCAR1/PYL9 (cloning via EcoR I and Xho I sites), pET28A-AtMYB44 (cloning via EcoR I and Xho I sites) and pET28A-ABI1 (cloning via BamH I and 
Hind III sites). For BiFC assay, RCAR1/PYL9 and AtMYB44 cDNAs with no stop codon were linked to the truncated YFP genes via $X b a$ I and Sal I sites in pUC-SPYCE and pUC-SPYNE vectors, respectively. For transient activation assay in protoplasts, $p R A B 18:: F L U C$ reporter plasmid has been described previously [5]. The effector plasmids are all derivatives of pBI221 [35]. The plasmids p35S::ABI1-GFP was created as described previously [36]; p35S::RCAR1/PYL9-HA and p35S::AtMYB44-myc constructs were generated by replacing the glucuronidase gene of pBI221 (via $X b a$ I and $S a c$ I sites) using the corresponding cDNA fragments; the internal control construct p35S::RLUC were produced as described [37]. Primers used in this study are listed in Table S2.

\subsection{Yeast Two-Hybrid Assay}

Arabidopsis GAL4 AD/cDNA library was constructed according to Matchmaker cDNA Synthesis protocol from Clontech Laboratories, Inc. Yeast two-hybrid screening was performed as described [38]. The $\alpha$-galactosidase activity was determined in AH109 yeast report cells as described in the Yeast Protocols Handbook from Clontech Laboratories, Inc. To test the effect of ABA treatment on the interaction between MYBs and RCARs/PYR1/PYLs, $10 \mu \mathrm{M}(+)$-ABA (hereafter referred to ABA) was added into the indicated medium.

\subsection{GST Pull-Down Assay}

GST, GST-RCAR1/PYL9, GST-AtMYB44 and His-RCAR1/PYL9, His-AtMYB44, His-ABI1 proteins were expressed in Escherichia coli and subsequently purified as described [6,39]. GST pull-down assay was performed as described [38]. For competitive pull-down assays, $5 \mu \mathrm{g}$ His-RCAR1/PYL9 with 0,5 or $25 \mu \mathrm{g}$ His-ABI1, or $25 \mu \mathrm{g}$ His protein were incubated with immobilized GST-AtMYB44 (5 $\mu \mathrm{g})$ in protein pull-down incubation buffer 2 (50 $\mathrm{mM}$ Tris- $\mathrm{HCl}$, $\mathrm{pH} 7.5,100 \mathrm{mM} \mathrm{NaCl}, 1 \mathrm{mM}$ EDTA, $0.05 \% \beta$-mercaptoethanol and $0.2 \%$ Triton X-100, $10 \mathrm{mM}$ $\mathrm{MgCl}_{2}$ ) at $4{ }^{\circ} \mathrm{C}$ for $2 \mathrm{~h}$. Proteins retained on the beads were resolved by SDS-PAGE and detected using anti-GST (Tiangen, Beijing, China) or anti-His antibody (Sigma, St. Louis, MO, USA), respectively. When indicated, $10 \mu \mathrm{M}$ ABA was added into the buffer.

\subsection{BiFC Assay}

BiFC assay was performed as described [40]. The pUC-SPYNE-AtMYB44 and pUC-SPYCE-RCAR1/ PYL9 constructs (containing control plasmids) were introduced into Arabidopsis protoplasts, as previously described [41]. Transfected protoplasts were collected $12 \mathrm{~h}$ after incubation and stained by DAPI as described [42]. The fluorescence signals of the DAPI-stained protoplasts were then observed and imaged by a Leica confocal laser-scanning microscope (Leica, Buffalo Grove, IL, USA).

\subsection{RNA Isolation and $q R T-P C R$ Analysis}

Total RNAs were extracted from 2-week-old plants of the wild-type and atmyb44 mutant plants using the RNAiso Plus reagent from Takara (Otsu, Japan). The cDNA was synthesized from $1 \mu \mathrm{g}$ of total RNAs with PrimeScript RT-PCR reagent Kit of Takara. qRT-PCR was performed using the CFX96 real-time PCR detection system from Bio-Rad Laboratories (Hercules, CA, USA) and 
SYBR Premix Ex Taq II from Takara (Otsu, Japan). The relative expression levels were calculated using the $\Delta \Delta C_{\mathrm{t}}$ method [43], which was normalized against the expression level of $A C T I N 2 / 8$. For all qRT-PCR analyses, three technical replicates and three independent biological repetitions were performed. Statistical significance was assessed by Student's $t$-test. Values of $p<0.05$ were considered significant. Primers used for this assay are listed Table S2.

\subsection{Protoplast Isolation and Transient Activation Assay}

Preparation of Arabidopsis protoplasts and subsequent transfection of protoplasts were performed as described above. The vector $p R A B 18:: F L U C$ was used at $2.5 \mu \mathrm{g}$ per transfection, $p 35 S: \because A t M Y B 44-M y c$, p35S::RCAR1/PYL9-HA and $p 35 S:: A B I 1-G F P$ were used at $2 \mu \mathrm{g}$ per transfection, and $p 35 S:: R L U C$ was used at $0.5 \mu \mathrm{g}$ per transfection. When indicated, $5 \mu \mathrm{M}$ ABA was added into the incubation buffer. Luciferase activity was measured by an LMax $\mathrm{II}^{384}$ luminometer (Molecular Devices, Bad Wildbad, Germany) using the Dual-Luciferase Reporter Assay System from Promega (Madison, WI, USA). Relative FLUC activity was calculated by normalizing against the $R L U C$ activity, and the data presented were the averages of three biological replicates.

\subsection{PP2C Enzyme Assay}

Proteins used in this assay were expressed and purified as described above. Phosphatase assay using pNPP as substrate was conducted as described [6] with minor modifications. Reaction mixtures $(500 \mu \mathrm{L})$ contained $50 \mathrm{mM}$ Tris- $\mathrm{HCl}(\mathrm{pH} 8.0), 150 \mathrm{mM} \mathrm{NaCl}, 10 \mathrm{mM} \mathrm{MgCl} 2,25 \mathrm{mM} \mathrm{pNPP}$, $0.5 \mu \mathrm{M}$ His-ABI1 with the different combinations of $5 \mu \mathrm{M}$ His-RCAR1/PYL9 or $10 \mu \mathrm{M}$ GST-AtMYB44 (or GST) and 0 or $10 \mu \mathrm{M}$ ABA. The release of PNP was measured at $410 \mathrm{~nm}, \mathrm{t} \sim 5 \mathrm{~s}$, intervals over $30 \mathrm{~min}$ and at $30{ }^{\circ} \mathrm{C}$ by a SpectraMAX M2 microplate reader (Molecular Devices, Sunnyvale, CA, USA). The values shown were normalized to the control (ABI1 only; $0 \mu \mathrm{M}$ ABA) and expressed as $\%$ of ABI1 activity.

\section{Conclusions}

In summary, we demonstrate that an R2R3-type MYB transcription factor, AtMYB44, interacts with the ABA receptor RCAR1/PYL9 and likely reduces its inhibitory effect on ABI1 activity in vitro and in vivo. These results may explain how AtMYB44 participates in ABA signaling.

\section{Acknowledgments}

This work was supported by grants from the National Natural Science Foundation of China (31171586 to Y.Y.; 31271758 to Jia.W.) and National 973 project (2013CB733903 to Y.Y.).

\section{Conflicts of Interest}

The authors declare no conflict of interest. 


\section{References}

1. Finkelstein, R.R.; Gampala, S.S.; Rock, C.D. Abscisic acid signaling in seeds and seedlings. Plant Cell 2002, 14, S15-S45.

2. Zheng, X.; Chen, B.; Lu, G.; Han, B. Overexpression of a NAC transcription factor enhances rice drought and salt tolerance. Biochem. Biophys. Res. Commun. 2009, 379, 985-989.

3. Cutler, S.R.; Rodriguez, P.L.; Finkelstein, R.R.; Abrams, S.R. Abscisic acid: Emergence of a core signaling network. Annu. Rev. Plant Biol. 2010, 61, 651-679.

4. Hubbard, K.E.; Nishimura, N.; Hitomi, K.; Getzoff, E.D.; Schroeder, J.I. Early abscisic acid signal transduction mechanisms: Newly discovered components and newly emerging questions. Genes Dev. 2010, 24, 1695-1708.

5. Ma, Y.; Szostkiewicz, I.; Korte, A.; Moes, D.; Yang, Y.; Christmann, A.; Grill, E. Regulators of PP2C phosphatase activity function as abscisic acid sensors. Science 2009, 324, 1064-1068.

6. Park, S.-Y.; Fung, P.; Nishimura, N.; Jensen, D.R.; Fujii, H.; Zhao, Y.; Lumba, S.; Santiago, J.; Rodrigues, A.; Chow, T.-F.F. Abscisic acid inhibits type $2 \mathrm{C}$ protein phosphatases via the PYR/PYL family of START proteins. Science 2009, 324, 1068-1071.

7. Santiago, J.; Rodrigues, A.; Saez, A.; Rubio, S.; Antoni, R.; Dupeux, F.; Park, S.Y.; Márquez, J.A.; Cutler, S.R.; Rodriguez, P.L. Modulation of drought resistance by the abscisic acid receptor PYL5 through inhibition of clade A PP2Cs. Plant J. 2009, 60, 575-588.

8. Saavedra, X.; Modrego, A.; Rodríguez, D.; González-García, M.P.; Sanz, L.; Nicolás, G.; Lorenzo, O. The nuclear interactor PYL8/RCAR3 of Fagus sylvatica FsPP2C1 is a positive regulator of abscisic acid signaling in seeds and stress. Plant Physiol. 2010, 152, 133-150.

9. Fuchs, S.; Grill, E.; Meskiene, I.; Schweighofer, A. Type $2 \mathrm{C}$ protein phosphatases in plants. FEBS J. 2012, 280, 681-693.

10. Yoshida, R.; Umezawa, T.; Mizoguchi, T.; Takahashi, S.; Takahashi, F.; Shinozaki, K. The regulatory domain of SRK2E/OST1/SnRK2. 6 interacts with ABI1 and integrates abscisic acid (ABA) and osmotic stress signals controlling stomatal closure in Arabidopsis. J. Biol. Chem. 2006, 281, 5310-5318.

11. Fujii, H.; Verslues, P.E.; Zhu, J.-K. Identification of two protein kinases required for abscisic acid regulation of seed germination, root growth, and gene expression in Arabidopsis. Plant Cell 2007, 19, 485-494.

12. Nakashima, K.; Fujita, Y.; Kanamori, N.; Katagiri, T.; Umezawa, T.; Kidokoro, S.; Maruyama, K.; Yoshida, T.; Ishiyama, K.; Kobayashi, M. Three Arabidopsis SnRK2 protein kinases, SRK2D/SnRK2. 2, SRK2E/SnRK2. 6/OST1 and SRK2I/SnRK2. 3, involved in ABA signaling are essential for the control of seed development and dormancy. Plant Cell Physiol. 2009, 50, 1345-1363.

13. Raghavendra, A.S.; Gonugunta, V.K.; Christmann, A.; Grill, E. ABA perception and signalling. Trends Plant Sci. 2010, 15, 395-401.

14. Yamaguchi-Shinozaki, K.; Shinozaki, K. Transcriptional regulatory networks in cellular responses and tolerance to dehydration and cold stresses. Annu. Rev. Plant Biol. 2006, 57, 781-803. 
15. Huang, D.; Jaradat, M.R.; Wu, W.; Ambrose, S.J.; Ross, A.R.; Abrams, S.R.; Cutler, A.J. Structural analogs of ABA reveal novel features of ABA perception and signaling in Arabidopsis. Plant J. 2007, 50, 414-428.

16. Jaradat, M.R.; Feurtado, J.A.; Huang, D.; Lu, Y.; Cutler, A.J. Multiple roles of the transcription factor AtMYBR1/AtMYB44 in ABA signaling, stress responses, and leaf senescence. BMC Plant Biol. 2013, 13, 192.

17. Jung, C.; Seo, J.S.; Han, S.W.; Koo, Y.J.; Kim, C.H.; Song, S.I.; Nahm, B.H.; Do Choi, Y.; Cheong, J.-J. Overexpression of AtMYB44 enhances stomatal closure to confer abiotic stress tolerance in transgenic Arabidopsis. Plant Physiol. 2008, 146, 623-635.

18. Persak, H.; Pitzschke, A. Tight interconnection and multi-level control of Arabidopsis MYB44 in MAPK cascade signalling. PLoS One 2013, 8, e57547.

19. Nguyen, X.C.; Hoang, M.H.T.; Kim, H.S.; Lee, K.; Liu, X.-M.; Kim, S.H.; Bahk, S.; Park, H.C.; Chung, W.S. Phosphorylation of the transcriptional regulator MYB44 by mitogen activated protein kinase regulates Arabidopsis seed germination. Biochem. Biophys. Res. Commun. 2012, 423, 703-708.

20. Stracke, R.; Werber, M.; Weisshaar, B. The R2R3-MYB gene family in Arabidopsis thaliana. Curr. Opin. Plant Biol. 2001, 4, 447-456.

21. Lång, V.; Palva, E.T. The expression of a rab-related gene, rab18, is induced by abscisic acid during the cold acclimation process of Arabidopsis thaliana (L.) Heynh. Plant Mol. Biol. 1992, 20,951-962.

22. Ghelis, T.; Dellis, O.; Jeannette, E.; Bardat, F.; Cornel, D.; Miginiac, E.; Rona, J.-P.; Sotta, B. Abscissic acid specific expression of $R A B 18$ involves activation of anion channels in Arabidopsis thaliana suspension cells. FEBS Lett. 2000, 474, 43-47.

23. Hou, X.; Lee, L.Y.C.; Xia, K.; Yan, Y.; Yu, H. DELLAs modulate jasmonate signaling via competitive binding to JAZs. Dev. Cell 2010, 19, 884-894.

24. Sánchez-Barrena, M.J.; Fujii, H.; Angulo, I.; Martínez-Ripoll, M.; Zhu, J.-K.; Albert, A. The structure of the $C$-terminal domain of the protein kinase AtSOS2 bound to the calcium sensor AtSOS3. Mol. Cell 2007, 26, 427-435.

25. Gosti, F.; Beaudoin, N.; Serizet, C.; Webb, A.A.; Vartanian, N.; Giraudat, J. ABI1 protein phosphatase $2 \mathrm{C}$ is a negative regulator of abscisic acid signaling. Plant Cell 1999, 11, 1897-1909.

26. Merlot, S.; Gosti, F.; Guerrier, D.; Vavasseur, A.; Giraudat, J. The ABI1 and ABI2 protein phosphatases $2 \mathrm{C}$ act in a negative feedback regulatory loop of the abscisic acid signalling pathway. Plant J. 2001, 25, 295-303.

27. Kranz, H.D.; Denekamp, M.; Greco, R.; Jin, H.; Leyva, A.; Meissner, R.C.; Petroni, K.; Urzainqui, A.; Bevan, M.; Martin, C. Towards functional characterisation of the members of the R2R3-MYB gene family from Arabidopsis thaliana. Plant J. 1998, 16, 263-276.

28. Nishimura, N.; Sarkeshik, A.; Nito, K.; Park, S.Y.; Wang, A.; Carvalho, P.C.; Lee, S.; Caddell, D.F.; Cutler, S.R.; Chory, J. PYR/PYL/RCAR family members are major in-vivo ABI1 protein phosphatase 2C-interacting proteins in Arabidopsis. Plant J. 2010, 61, 290-299.

29. Léon-Kloosterziel, K.M.; Gil, M.A.; Ruijs, G.J.; Jacobsen, S.E.; Olszewski, N.E.; Schwartz, S.H.; Zeevaart, J.A.; Koornneef, M. Isolation and characterization of abscisic acid-deficient Arabidopsis mutants at two new loci. Plant J. 1996, 10, 655-661. 
30. Shin, R.; Burch, A.Y.; Huppert, K.A.; Tiwari, S.B.; Murphy, A.S.; Guilfoyle, T.J.; Schachtman, D.P. The Arabidopsis transcription factor MYB77 modulates auxin signal transduction. Plant Cell 2007, 19, 2440-2453.

31. Liu, R.; Chen, L.; Jia, Z.; Lü, B.; Shi, H.; Shao, W.; Dong, H. Transcription factor AtMYB44 regulates induced expression of the Ethylene insensitive2 gene in Arabidopsis responding to a harpin protein. Mol. Plant-Microbe Interact. 2011, 24, 377-389.

32. Shim, J.S.; Jung, C.; Lee, S.; Min, K.; Lee, Y.W.; Choi, Y.; Lee, J.S.; Song, J.T.; Kim, J.K.; Choi, Y.D. AtMYB44 regulates WRKY70 expression and modulates antagonistic interaction between salicylic acid and jasmonic acid signaling. Plant J. 2013, 73, 483-495.

33. Persak, H.; Pitzschke, A. Dominant repression by Arabidopsis transcription Factor MYB44 causes oxidative damage and hypersensitivity to abiotic stress. Int. J. Mol. Sci. 2014, 15, 2517-2537.

34. Murashige, T.; Skoog, F. A revised medium for rapid growth and bio assays with tobacco tissue cultures. Physiol. Plant 1962, 15, 473-497.

35. Jefferson, R.A. Assaying chimeric genes in plants: The Gus gene fusion system. Plant Mol. Biol. Report. 1987, 5, 387-405.

36. Moes, D.; Himmelbach, A.; Korte, A.; Haberer, G.; Grill, E. Nuclear localization of the mutant protein phosphatase abil is required for insensitivity towards ABA responses in Arabidopsis. Plant J. 2008, 54, 806-819.

37. Licausi, F.; Weits, D.A.; Pant, B.D.; Scheible, W.R.; Geigenberger, P.; van Dongen, J.T. Hypoxia responsive gene expression is mediated by various subsets of transcription factors and miRNAs that are determined by the actual oxygen availability. New Phytol. 2011, 190, 442-456.

38. Li, D.; Wang, X.; Yuan, D.; Zhang, L.; Jiang, X.; Tao, Z.; Li, Y.; Wang, J.; Li, X.; Yang, Y. Over-expression of ArathEULS3 confers ABA sensitivity and drought tolerance in Arabidopsis. Plant Cell Tissue Organ 2014, doi:10.1007/s11240-014-0453-0.

39. Yang, Y.; Sulpice, R.; Himmelbach, A.; Meinhard, M.; Christmann, A.; Grill, E. Fibrillin expression is regulated by abscisic acid response regulators and is involved in abscisic acid-mediated photoprotection. Proc. Natl. Acad. Sci. USA 2006, 103, 6061-6066.

40. Walter, M.; Chaban, C.; Schütze, K.; Batistic, O.; Weckermann, K.; Näke, C.; Blazevic, D.; Grefen, C.; Schumacher, K.; Oecking, C. Visualization of protein interactions in living plant cells using bimolecular fluorescence complementation. Plant J. 2004, 40, 428-438.

41. Yoo, S.-D.; Cho, Y.-H.; Sheen, J. Arabidopsis mesophyll protoplasts: A versatile cell system for transient gene expression analysis. Nat. Protoc. 2007, 2, 1565-1572.

42. Shen, G.; Kuppu, S.; Venkataramani, S.; Wang, J.; Yan, J.; Qiu, X.; Zhang, H. Ankyrin repeat-containing protein $2 \mathrm{a}$ is an essential molecular chaperone for peroxisomal membrane-bound Ascorbate peroxidase3 in Arabidopsis. Plant Cell 2010, 22, 811-831.

43. Livak, K.J.; Schmittgen, T.D. Analysis of relative gene expression data using real-time quantitative PCR and the $2^{-\Delta \Delta C t}$ method. Methods 2001, 25, 402-408.

(C) 2014 by the authors; licensee MDPI, Basel, Switzerland. This article is an open access article distributed under the terms and conditions of the Creative Commons Attribution license (http://creativecommons.org/licenses/by/3.0/). 